\title{
Performance Comparison of GeneXpert MTB/ RIF and ProbeTec ET Tests for Rapid Molecular Diagnosis of Extrapulmonary Tuberculosis in a Low TB/MDR-TB Incidence Country
}

\author{
Eiman Mokaddas ${ }^{\mathrm{a}, \mathrm{b}}$ Suhail Ahmad ${ }^{\mathrm{a}}$ Hanaa Eldeen $^{\mathrm{b}}$

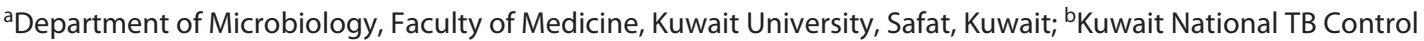 \\ Laboratory, Shuwaikh, Kuwait
}

\section{Highlights of the Study}

- GeneXpert MTB/RIF (Xpert) detected extrapulmonary tuberculosis (EPTB) in more specimens compared to ProbeTec ET (PTec-ET) in smear-positive and culture-positive samples.

- Xpert also detected EPTB in more specimens with histopathological evidence of tuberculosis (TB) and in cerebrospinal fluids for diagnosing TB meningitis.

- Xpert will help in better management of patients with EPTB, particularly in countries with low incidence of TB where EPTB is more common.

\section{Keywords}

Mycobacterium tuberculosis · Extrapulmonary tuberculosis · Molecular diagnosis · Xpert MTB/RIF assay · Probe Tec ET test · Kuwait

\begin{abstract}
Objective: This study evaluated the performance of GeneXpert MTB/RIF (Xpert) and ProbeTec ET (PTec-ET) assays in diagnosing extrapulmonary tuberculosis (EPTB) in Kuwait. Materials and Methods: We tested nonrespiratory clinical specimens ( $n=3,995)$ collected from 3,995 patients suspected to have EPTB. These included cavitary fluids ( $n=2,054)$, fineneedle aspirate (FNA)/pus/tissue biopsy ( $n=1,461)$, urine $(n=302)$, cerebrospinal fluid (CSF, $n=118)$, and others $(n=$ $60)$. All specimens were processed for acid-fast bacilli (AFB), culture in mycobacteria growth indicator tube 960 system,
\end{abstract}

karger@karger.com www.karger.com/mpp

Karger $\stackrel{\text { ' }}{5}$

BOPEN ACCESS
(C) 2021 The Author(s)

Published by S. Karger AG, Basel

This is an Open Access article licensed under the Creative Commons Attribution-NonCommercial-4.0 International License (CC BY-NC) (http://www.karger.com/Services/OpenAccessLicense), applicable to the online version of the article only. Usage and distribution for commercial purposes requires written permission. and nucleic acid detection by Xpert and PTec-ET according to manufacturer's instructions. Results: Of 3,995 specimens, 95 were AFB-positive, 403 were culture-positive, and an additional 86 samples had histopathology suggestive of TB. Using culture as reference, the sensitivity and specificity values were 88.33 and $97.3 \%$ for Xpert and 72.95 and $97.80 \%$ for PTec-ET, respectively. Although performance of both tests was comparable in AFB-positive samples, Xpert detected significantly more cases in culture-positive samples. Among culture-negative samples, Xpert detected 18 more cases including 16 with histopathological evidence of TB. Lowest positivity was detected for both tests in cavitary fluids. Xpert performed better than PTec-ET in culture-positive FNA/pus/ tissue biopsy and CSF samples. Conclusions: Although performance of both tests was suboptimal for AFB-negative/ culture-positive samples, Xpert performed better than PTecET and also detected more cases of AFB-negative/culture- 
negative/histopathology-positive samples. PTec-ET was positive in 3, while Xpert was positive in all 6 culture-positive CSF specimens for rapid diagnosis of TB meningitis. Xpert was thus superior to PTec-ET or smear microscopy in rapid diagnosis of EPTB.

(c) 2021 The Author(s)

Published by S. Karger AG, Basel

\section{Introduction}

Although the global incidence of tuberculosis (TB) and TB deaths is slowly declining, TB is still a formidable public health challenge. According to recent data released by the WHO, an estimated 10 million new active TB disease cases and $\sim 1.41$ million deaths occurred in 2019, making TB one of the top 10 causes of death worldwide and the leading cause of death from a single infectious agent [1]. Furthermore, an estimated 465,000 people developed TB that was resistant to rifampicin, and of these, nearly 363,000 (78\%) were multidrug-resistant (MDR)TB cases (defined as infection with a Mycobacterium tuberculosis strain resistant at least to rifampicin and isoniazid, the 2 most effective first-line drugs) which are much more difficult to treat than drug-susceptible TB [1-3]. Early detection of M. tuberculosis in clinical specimens, its susceptibility to anti-TB drugs, particularly rifampicin and effective treatment are essential for global TB control efforts [2-4].

Sputum smear-positive pulmonary $\mathrm{TB}$ is contagious as it spreads to close contacts, usually by coughing [5]. Extrapulmonary TB (EPTB) affects other parts of the body, mostly involving the pleura and lymph node, and is typically not infectious [6]. EPTB accounts for $\sim 15 \%$ of all TB cases worldwide with the maximum prevalence of $24 \%$ reported from the WHO Eastern Mediterranean Region $[1,7]$. The Eastern Mediterranean Region includes Kuwait, an Arabian Gulf country where 95\% of mycobacterial infections are caused by M. tuberculosis [8]. Kuwait has a low incidence of TB ( $\sim 24$ cases per 100,000 population), high (44\%) prevalence of EPTB, and a low ( 1.1\%) incidence of MDR-TB [7, 9]. Other studies from nearby countries (Saudi Arabia and Turkey) have also reported high numbers of EPTB cases $[10,11]$.

The diagnosis of EPTB is problematic as it often involves invasive procedures that require special expertise and is limited in accuracy and time to diagnosis $[6,12]$. Although culture is the gold standard for the diagnosis of active TB, it has low sensitivity (30-50\%) for pleural fluid and even lower for cerebrospinal fluid (CSF) $[4,6,12,13]$. Culture is also sometimes negative for biopsy material even when there is histopathological evidence suggestive of active TB disease [6, 12, 14-16].

The GeneXpert MTB/RIF (Xpert) assay has been endorsed by the WHO as a rapid molecular test for the diagnosis of pulmonary TB in low- and middle-income countries and allows the simultaneous detection of $M$. $t u$ berculosis complex and the most common mutations in the $r p o B$ gene conferring resistance to rifampicin in $<2 \mathrm{~h}$ $[1,17]$. However, the performance of Xpert assay in nonrespiratory samples for the diagnosis of EPTB is highly variable ranging from $83-96 \%$ in lymph node tissue and aspirates to $34-46 \%$ in pleural fluid with smear-positive samples yielding higher sensitivities [14-16]. Similarly, the diagnostic performance of ProbeTec ET (PTec-ET) assay with nonrespiratory samples is not well established [18]. This study was performed to evaluate the performance of Xpert and PTec-ET tests in diagnosing EPTB in Kuwait, an Arabian Gulf country with a low incidence of TB and MDR-TB [7].

\section{Materials and Methods}

Patients, Specimens, and M. tuberculosis Isolates

A total of 3,995 nonrespiratory specimens collected from 3,995 suspected TB patients during November 2011-November 2015 at Kuwait National TB Control Laboratory were used in this retrospective study. The clinical specimens were collected after obtaining verbal consent from suspected TB patients as part of routine diagnostic work-up and resistance surveillance, and the results are reported on de-identified samples. All samples were processed for smear microscopy for acid-fast bacilli (AFB), culture on liquid medium by mycobacteria growth indicator tube (MGIT) 960 system and detection of M. tuberculosis DNA with/without additional detection of resistance to rifampicin by Xpert and PTec-ET assays.

The smears for detection of AFB were prepared by ZiehlNeelsen stain and the cultures from nonsterile samples were processed by using $\mathrm{N}$-acetyl-L-cysteine and sodium hydroxide (NALC/NaOH), while sterile clinical specimens were directly processed according to the instructions supplied by the manufacturer of MGIT 960 system (Becton Dickinson, Sparks, MD, USA) and as described previously $[9,19]$. The cultures flagged positive by MGIT 960 system were identified as $M$. tuberculosis by using the AccuProbe DNA probes, as described previously $[9,19]$. All $M$. tuberculosis positive cultures were subjected to phenotypic drug susceptibility testing (DST) against 4 (streptomycin, isoniazid, rifampicin, and ethambutol) drugs (final drug concentrations: 1.0 $\mathrm{mg} / \mathrm{L}$ for streptomycin, $0.1 \mathrm{mg} / \mathrm{L}$ for isoniazid, $1.0 \mathrm{mg} / \mathrm{L}$ for rifampicin, and $5.0 \mathrm{mg} / \mathrm{L}$ for ethambutol) by MGIT 960 system, as described previously $[9,19]$.

Cepheid GeneXpert MTB/RIF and ProbeTec ET Assays

The Xpert (Cepheid, Sunnyvale, CA, USA) assay was performed directly on clinical samples without prior extraction, and the results were interpreted by using the GeneXpert MTB/RIF cartridge and software according to the manufacturer's instructions and as de- 
Table 1. Comparison of GeneXpert MTB/RIF (Xpert) and ProbeTec ET (PTec-ET) test results with smear microscopy for AFB and MGIT 960 system culture among 3,995 extrapulmonary specimens

\begin{tabular}{|c|c|c|c|c|}
\hline \multirow[t]{2}{*}{ Molecular test result } & \multicolumn{2}{|c|}{$\begin{array}{l}\text { MGIT } 960 \text { system culture- } \\
\text { positive }\end{array}$} & \multicolumn{2}{|c|}{$\begin{array}{l}\text { MGIT } 960 \text { system culture- } \\
\text { negative }\end{array}$} \\
\hline & AFB-positive & AFB-negative & AFB-positive & AFB-negative \\
\hline Xpert-positive/PTec-ET-positive & 74 & 216 & $17^{\mathrm{a}}$ & $60^{\mathrm{b}}$ \\
\hline Xpert-positive/PTec-ET-negative & 2 & 64 & 1 & $19^{c}$ \\
\hline Xpert-negative/PTec-ET-positive & 0 & 4 & 0 & 2 \\
\hline Xpert-negative/PTec-ET-negative & 1 (NTM) & 42 & 0 & 3,493 \\
\hline All Xpert-positive only & 76 & 280 & 18 & 79 \\
\hline All PTec-ET-positive only & 74 & 220 & 17 & 62 \\
\hline$\kappa$ & 0.49 & 0.44 & 0 & 0.848 \\
\hline$\kappa$ & 0.46 & & 0.87 & \\
\hline$\kappa$ & 0.875 & & & \\
\hline
\end{tabular}

MGIT, mycobacteria indicator growth tube; AFB, acid-fast bacilli; NTM, nontuberculous mycobacteria; $\kappa$, kappa coefficient. ${ }^{a} 16$ samples showed histopathological evidence of TB. ${ }^{\mathrm{b}} 54$ samples showed histopathological evidence of TB. ${ }^{c} 16$ samples showed histopathological evidence of TB.

scribed previously [20]. The PTec-ET (Becton Dickinson, Sparks, $\mathrm{MD}$, USA) assay was also performed by following the manufacturer's instructions and as described previously [21]. Briefly, the DNA released from decontaminated samples $(150 \mu \mathrm{L})$ in duplicate was added to the priming wells of the microtiter plate containing primers and probes to rehydrate the reagents, and then $100 \mu \mathrm{L}$ of the priming mix was added to the corresponding wells of another microtiter plate containing activated enzymes, buffer, and dNTPs to initiate amplification in the BD ProbeTec analyzer. Positive and negative controls were run with each assay including internal controls to avoid false-positive and false-negative results due to amplification inhibitors in clinical specimens and the results were interpreted according to the manufacturer's instructions.

\section{Detection of Rifampicin Resistance by GenoType MTBDRplus} Assay

Culture isolates of M. tuberculosis grown from clinical samples exhibiting rifampicin resistance by Xpert assay were also tested by reverse hybridization-based GenoType MTBDRplus assay (Hain Lifescience, Nehren, Germany) which detects resistance to rifampicin as well as to isoniazid [2]. Ten rifampicin-susceptible $M$. tuberculosis isolates were also tested for comparison. Genomic DNA from the MGIT 960 system cultures was prepared by using Chelex-100, as described previously [22]. The manual GenoType MTBDRplus assay was performed, and the results were interpreted according to the manufacturer's recommendations and as described in detail previously [23]. Negative controls (water instead of DNA) were included in each run. The GenoType MTBDRplus assay results were also confirmed by PCR amplification followed by DNA sequencing (PCR-sequencing) of hot-spot region of the $r p o B$ and $k a t G$ codon 315 regions, performed as described previously [19, 23].

\section{Statistical Analyses}

Categorical variables were expressed as absolute number. Statistical analysis was performed using Fisher's exact test and probability levels $<0.05$ by the 2 -tailed test were considered as statisti- cally significant. Statistical analyses were performed using WinPepi software ver. 3.8 (PEPI for Windows, Microsoft Inc., Redmond, WA, USA) or GraphPad software (GraphPad, La Jolla, CA, USA). The strength of agreement between the different test results was assessed by using the robust kappa statistics. A kappa coefficient $(\kappa)$ value of $<0.2,0.21-0.4,0.41-0.6,0.61-0.8$, and $0.81-1.0$ indicated slight agreement, fair agreement, moderate agreement, substantial agreement, and almost perfect agreement, respectively [24].

\section{Results}

\section{Clinical Specimens}

3,995 extrapulmonary specimens collected from 3,995 suspected TB patients were used. Clinical samples, obtained before initiation of anti-TB therapy, included cavitary fluids (including pleural fluid, $n=1,340$; ascitic fluid, $n=484$; pericardial fluid, $n=45$, and other body fluids, $n=185$ ); fine-needle aspirate (FNA), $n=668$; pus, $n=$ 444; tissue biopsies, $n=349$; urine, $n=302$; CSF, $n=118$; and other miscellaneous specimens including stools, swabs etc., $n=60$. FNA samples were obtained from lymph nodes, but the source of tissue biopsies and pus samples were not available. Skin pus swabs were included among other miscellaneous specimens.

\section{Smear Microscopy, MGIT 960 System Cultures, Xpert, and PTec-ET Tests}

The results for AFB and MGIT 960 system culture positivity and for molecular detection of $M$. tuberculosis by Xpert and PTec-ET are presented in Table 1. 95 samples 
Table 2. Positivity, sensitivity, specificity, PPV, and NPV of GeneXpert MTB/RIF (Xpert) and ProbeTec ET (PTec-ET) tests for the diagnosis of extrapulmonary TB with MGIT 960 system culture or CRS as a reference standard

\begin{tabular}{lllllll}
\hline Reference & Molecular test & Positives, $n$ & Sensitivity, $\%$ & Specificity, \% & PPV, * $\%$ & NPV, * \% \\
\hline Culture* $(n=403)$ & Xpert & 356 & 88.33 & 97.30 & 65.56 & 98.67 \\
& PTec-ET & 294 & 72.95 & 97.80 & 78.82 & 96.99 \\
\hline CRS* $^{*}(n=489)$ & Xpert & 442 & 90.39 & 99.69 & 95.57 & 98.67 \\
& PTec-ET & 364 & 74.44 & 99.74 & 97.59 & 96.55 \\
\hline
\end{tabular}

PPV, positive predictive value; NPV, negative predictive value; MGIT, mycobacteria growth indicator tube. * Culture, MGIT 960 culture; CRS which included MGIT 960 culture and/or clinical diagnosis of TB.

were positive for AFB by microscopy and 403 specimens yielded mycobacterial growth (including 1 nontuberculous mycobacteria, NTM culture) by MGIT 960 system. Among 3,592 culture-negative specimens, 86 samples exhibited evidence of histopathological TB including 18 AFB-positive samples.

Among 95 AFB-positive specimens, Xpert was positive in $94(98.95 \%)$ samples, while PTec-ET was positive in 90 (94.74) samples, but the difference was not significant $(p=0.211)$. However, among 403 culture-positive specimens, Xpert and PTec-ET detected M. tuberculosis in 356 (88.34\%) and 294 (72.95\%) specimens, respective$l y$, and the difference was statistically significant $(p=$ 0.001). The performance of both Xpert and PTec-ET was nearly same in AFB-positive, culture-positive and AFBpositive, culture-negative specimens (Table 1). However, in AFB-negative, culture-positive specimens $(n=326)$, Xpert and PTec-ET detected M. tuberculosis in 280 $(85.89 \%)$ and $220(67.48 \%)$ samples, respectively, and the difference was statistically significant $(p=0.001)$. Although Xpert also detected M. tuberculosis in 79 (2.21\%) samples while PTec-ET detected it in $62(1.73 \%)$ samples among 3,574 AFB-negative, culture-negative specimens (including 70 samples with histopathological evidence of $\mathrm{TB})$, the difference did not reach statistical significance $(p=0.148)$ (Table 1).

Using culture as the gold standard, 356 (88.34\%) samples were positive by Xpert among 403 culture-positive samples yielding a sensitivity, specificity, positive predictive value (PPV), and negative predictive value (NPV) of $88.34,97.30,65.56$, and $98.67 \%$, respectively, for the Xpert assay (Table 2). The sensitivity, specificity, PPV, and NPV for the PTec-ET test were 72.95, 97.80, 78.82, and $96.99 \%$, respectively (Table 2). The difference in the sensitivity values by the 2 tests was statistically significant $(p=0.001)$. Using a composite reference standard (CRS) that included laboratory (culture) and/or clinical (histopathological evidence of infection) diagnosis of TB ( $n=$ 489 ), the sensitivity of Xpert was also higher (90.39\% positive) than PTec-ET (364 positives among 489 samples, $74.44 \%)(p=0.001)$. The specificity, PPV, and NPV values against CRS were $99.69,95.57$, and $98.67 \%$ for Xpert and 99.74, 97.59, and 96.55\% for PTec-ET, respectively (Table 2). The concordance between Xpert and PTec-ET test results was examined by calculating $\kappa$ values; the data showed almost perfect agreement $(\kappa, 0.87)$ among all samples. However, only moderate agreement $(\kappa, 0.46)$ was seen among MGIT culture-positive samples, while almost perfect agreement $(\kappa, 0.87)$ was obtained for culture-negative samples.

Performance Comparison of Xpert and PTec-ET Tests in Different Extrapulmonary Specimens

The results for the detection of $M$. tuberculosis by Xpert and PTec-ET in various categories of clinical specimens are presented in Table 3. Among culturepositive samples, Xpert detected M. tuberculosis in more samples than PTec-ET in cavitary fluids, urine, CSF, and other miscellaneous specimens, however, the differences were not statistically significant. On the contrary, in FNA/pus/tissue biopsy specimens, Xpert, and PTec-ET detected M. tuberculosis in 283 (93.09\%) and $233(76.64 \%)$ samples and the difference was statistically significant $(p=0.001)$ (Table 3 ). Against culture, the sensitivity and specificity values for cavitary fluids, FNA/pus/tissue biopsy, urine, CSF and other (stool/ swab, etc.) specimens were 69.7 and $99.6 \%, 93.1$ and $92.3 \%, 88.9$ and $100 \%, 100$ and $100 \%$, and 75 and $100 \%$, respectively, for Xpert and 64.5 and $99.7 \%, 76.6$ and $93.8 \%, 77.8$ and $100 \%, 50$ and $100 \%$, and 25 and $100 \%$, 
Table 3. Comparison of GeneXpert MTB/RIF (Xpert) and ProbeTec ET (PTec-ET) test results with MGIT 960 system culture among various different extrapulmonary specimens

\begin{tabular}{|c|c|c|c|c|c|c|c|c|c|c|}
\hline \multirow[t]{2}{*}{ Molecular test result } & \multicolumn{2}{|c|}{$\begin{array}{l}\text { Cavitary fluids, } n= \\
2,054\end{array}$} & \multicolumn{2}{|c|}{$\begin{array}{l}\text { FNA/pus/tissue } \\
\text { biopsy, } n=1,461\end{array}$} & \multicolumn{2}{|c|}{ Urine, $n=302$} & \multicolumn{2}{|c|}{ CSF, $n=118$} & \multicolumn{2}{|c|}{$\begin{array}{l}\text { Others (stool and } \\
\text { swabs), } n=60\end{array}$} \\
\hline & $\begin{array}{l}\text { culture- } \\
\text { positive }\end{array}$ & $\begin{array}{l}\text { culture- } \\
\text { negative }\end{array}$ & $\begin{array}{l}\text { culture- } \\
\text { positive }\end{array}$ & $\begin{array}{l}\text { culture- } \\
\text { negative }\end{array}$ & $\begin{array}{l}\text { culture- } \\
\text { positive }\end{array}$ & $\begin{array}{l}\text { culture- } \\
\text { negative }\end{array}$ & $\begin{array}{l}\text { culture- } \\
\text { positive }\end{array}$ & $\begin{array}{l}\text { culture- } \\
\text { negative }\end{array}$ & $\begin{array}{l}\text { culture- } \\
\text { positive }\end{array}$ & $\begin{array}{l}\text { culture- } \\
\text { negative }\end{array}$ \\
\hline Xpert-positive/PTec-ET-positive & 47 & 6 & 231 & $70^{\mathrm{a}}$ & 7 & 0 & 3 & 0 & 2 & 1 \\
\hline Xpert-positive/PTec-ET-negative & 6 & 1 & 52 & $19^{\mathrm{b}}$ & 1 & 0 & 3 & 0 & 4 & 0 \\
\hline Xpert-negative/PTec-ET-positive & 2 & 0 & 2 & 2 & 0 & 0 & 0 & 0 & 0 & 0 \\
\hline Xpert-negative/PTec-ET-negative & 21 & 1,971 & $19(1 \mathrm{NTM})$ & 1,066 & 1 & 293 & 0 & 112 & 2 & 51 \\
\hline Xpert/culture positivity, \% & $53(69.7)$ & na & $283(93.1)$ & na & $8(88.9)$ & na & $6(100)$ & na & $6(75)$ & na \\
\hline PTec-ET/culture positivity, $\%$ & $49(64.4)$ & na & $233(76.6)$ & na & $7(77.7)$ & na & $3(50)$ & na & $2(25)$ & na \\
\hline$\kappa$ coefficient & 0.76 & 0.92 & 0.34 & 0.87 & 0.61 & No value & 0 & No value & 0.2 & 1 \\
\hline$\kappa$ coefficient & 0.92 & & 0.86 & & 0.93 & & 0.66 & & 0.57 & \\
\hline
\end{tabular}

FNA, fine-needle aspirate; NTM, nontuberculous mycobacteria; na, not applicable; CSF, cerebrospinal fluid; TB, tuberculosis; MGIT, mycobacteria growth indicator tube. ${ }^{\mathrm{a}}$ All 70 samples showed histopathological evidence of TB. ${ }^{\mathrm{b}} 16$ samples showed histopathological evidence of TB.

respectively, for PTec-ET. Against CRS, the sensitivity and specificity values for FNA/pus/tissue biopsy specimens were 94.6 and $99.7 \%$, respectively, for Xpert and 77.7 and $99.8 \%$, respectively, for PTec-ET. Only fair agreement $(\kappa, 0.34)$ was apparent between Xpert and PTec-ET tests for culture-positive FNA/pus/tissue biopsy specimens, while substantial agreement was noted for culture-positive cavitary fluids $(\kappa, 0.76)$ and urine specimens $(\kappa, 0.61)$ (Table 3$)$. Among all categories of culture-negative samples, Xpert detected $M$. tuberculosis in more samples than PTec-ET; however, the differences were not statistically significant, and both tests exhibited almost perfect agreement for most sample types ( $\kappa$ range, 0.87-1.00). Overall, almost perfect agreement was seen between Xpert and PTec-ET for cavitary fluids, FNA/pus/tissue biopsy and urine specimens ( $\kappa$ range, 0.86-0.93), while lower agreement was seen for CSF and other specimens (Table 3 ).

\section{Phenotypic and Molecular Detection of Resistance to Rifampicin}

Phenotypic DST data showed that only one M. tuberculosis isolate was resistant to rifampicin, while the remaining 401 (excluding nontuberculous mycobacteria) isolates were rifampicin-susceptible. The Xpert results were concordant with phenotypic DST data. The GenoType MTBDRplus assay and PCR-sequencing studies performed on rifampicin-resistant and 10 selected rifampicin-susceptible isolates showed the presence of S456W mutation in the $r p o B$ gene in rifampicin-resistant isolate (and S315T mutation in kat $G$ conferring resistance to iso- niazid; MDR-TB) and wild-type $r p o B$ sequence among all 10 rifampicin-susceptible isolates. Interestingly, the culture-positive FNA sample, testing positive for M. tuberculosis and its resistance to rifampicin by Xpert, tested negative by PTec-ET assay.

\section{Discussion}

Rapid diagnosis of TB prevents the development of drug resistance and improves outcome, while delayed diagnosis increases illness and death, and incorrect diagnosis causes unnecessary treatment [1]. Routine tests for the diagnosis of EPTB are limited in sensitivity, accuracy, and time to diagnosis typically due to paucibacillary load $[6,12-16]$. Although culture is often used as the gold standard for the definitive diagnosis of TB, it has low sensitivity for specimens from extrapulmonary sites, particularly in some types of specimens (e.g., cavitary fluids) $[6,12]$. In some extrapulmonary samples with clear histopathological evidence of clinical TB disease, the culture remains negative $[14,16,25]$. Thus, some studies have used a combination of culture and clinical diagnosis of TB [25], or a CRS that includes a molecular diagnostic test, histology, smear, biochemical testing results, signs, and symptoms, or a response to treatment with anti-TB therapy in addition to culture [14]. Xpert has been approved for diagnosis of $\mathrm{TB}$ in respiratory specimens (particularly sputum samples); it has also been used with extrapulmonary specimens (off-label use) mainly due to limitations of other available tests for the diagnosis of 
EPTB [14-16]. Similarly, PTec-ET is also approved only for respiratory specimens; however, some studies have also evaluated its performance with nonrespiratory specimens [14, 21, 26, 27].

We performed a head-to-head comparison of the performance of Xpert and PTec-ET tests for the diagnosis of EPTB using a large number $(n=3,995)$ of extrapulmonary samples and culture or a CRS comprising culture and/or histopathological evidence of TB as reference. The sensitivity values with respect to smear-positive samples for Xpert and PTec-ET were 98.9 and 94.7\%, respectively. With culture as reference, the sensitivity, specificity, PPV, and NPV for the diagnosis of all forms of EPTB were 88.34, 97.30, 65.56, and 98.67\%, respectively, for Xpert and $72.95,97.80,78.82$, and $96.99 \%$, respectively, for PTec-ET. Similarly, the sensitivity with respect to CRS was also higher for Xpert (90.39\%) than PTec-ET (74.44\%). Again, the specificity values for both the tests were nearly same $(99.69 \%$ for Xpert vs. $99.74 \%$ for PTecET). Interestingly, using CRS as reference, the PPV for the diagnosis of EPTB for both Xpert (95.57\%) and PTecET (97.59\%) were nearly same. On the contrary, the PPV was relatively much lower for Xpert $(65.56 \%)$ than for PTec-ET (78.82\%) when culture was used as reference. This is largely due to the detection of several culture-negative specimens with histopathological evidence of active TB disease testing positive by Xpert assay. This phenomenon has also been reported previously with extrapulmonary samples in previous studies $[14,16,25]$. Only one previous study has performed head-to-head comparison of these 2 rapid molecular tests for the diagnosis of EPTB with a small number $(n=23)$ of nonrespiratory samples, and the sensitivity of Xpert observed to be higher (91\%) than PTec-ET (65\%) with culture as reference [18]. A few studies have also tested nonrespiratory specimens by PTec-ET and reported sensitivity values of $61.1 \%$ [21], $71.4 \%$ [26], or $100 \%$ [27] for the diagnosis of EPTB compared to culture. On the contrary, the Xpert has been extensively evaluated with nonrespiratory samples for the diagnosis of EPTB.

In an early large-scale study, the sensitivity and specificity of Xpert for the diagnosis of EPTB were reported as 81.3 and $99.8 \%$ against a reference standard that comprised culture and/or clinical diagnosis of TB [25]. Denkinger et al. [14] performed a meta-analysis of 18 studies based on 4,461 nonrespiratory samples and reported pooled Xpert sensitivity and specificity values against culture or a CRS comprising culture or a molecular diagnostic test (other than Xpert), histology, smear microscopy, biochemical testing results, clinical diagnosis, or a response to treatment with anti-TB therapy. Their data showed that the sensitivity varied greatly among different types of specimens making overall estimates of accuracy difficult; however, the pooled sensitivity across all specimen types was $97.4 \%$ against smear-positive samples [14]. Our results (Xpert sensitivity of $98.9 \%$ against smear-positive specimens) are thus in close agreement with the metadata reported by Denkinger et al. [14]. Thus, Xpert can rapidly differentiate EPTB from other mycobacterial infections in smear-positive specimens.

In a more recent review based on 66 unique studies (50 studies carried out in low-/middle-income countries) and based on 16,213 specimens, Kohli et al. [16] have also concluded that pooled Xpert sensitivity varies greatly across different specimen types, varying from a low of $31 \%$ in pleural fluid to $97 \%$ in bone or joint fluid against culture. However, the specificity values, as in our study, were very high (range 79-99\%) [16].

The data for various nonrespiratory specimens also showed that Xpert detected M. tuberculosis in more samples than PTec-ET including cavitary fluids, FNA/pus/ tissue biopsy, urine, CSF, and other miscellaneous specimens, with reference to both, culture or CRS as reference. The Xpert test showed a sensitivity of only $69.7 \%$ for cavitary fluids against culture but a higher sensitivity of 93.1\% for FNA/pus/tissue biopsy specimens against culture or $94.6 \%$ against CRS, while PTec-ET yielded a sensitivity of $64.5 \%$ for cavitary fluids and $76.6 \%$ for FNA/ pus/tissue biopsy specimens against culture or $77.7 \%$ for FNA/pus/tissue biopsy specimens against CRS. A metaanalysis of Xpert data has also shown lower pooled sensitivity in cavitary fluids that varied from $50.9 \%$ in pleural fluid (27 studies, 4,006 specimens) to $59.2 \%$ in peritoneal fluid (16 studies and 712 specimens) to $65.7 \%$ in pericardial fluid (7 studies and 324 specimens) against culture [16]. Also, pooled Xpert sensitivity was higher among FNA/pus/tissue biopsy specimens which varied from 97.2\% in bone or joint aspirates (5 studies, 385 specimens) to $91.8 \%$ in bone or joint tissue (7 studies and 618 specimens) to $87.6 \%$ in lymph node aspirate (17 studies and 1,710 specimens) to $78.9 \%$ in lymph node tissue (10 studies and 484 specimens) against culture [16]. Lower sensitivity (45\%) was also obtained for cavitary fluid (pleural fluid, $n=47$; ascitic fluid, $n=18$; and other sterile fluids, $n=17$ ) samples, while higher sensitivity of $72 \%$ was reported for FNA/pus specimens against culture in 1 PTec-ET study that tested a large number of nonrespiratory specimens [21]. Other studies using PTec-ET test have either used small number of extrapulmonary speci- 
mens $[18,26]$ or the data were not tabulated for individual specimen types [27].

Although the number of positive samples was relatively low, the sensitivity of Xpert was very high for CSF (100\%) and urine (89\%) specimens with $100 \%$ specificity against culture in our study. Kohli et al. [16] have also reported a pooled sensitivity of $71.1 \%$ and specificity of $98 \%$ in CSF (29 studies and 3,774 specimens) and a pooled sensitivity of $82.7 \%$ and specificity of $98.7 \%$ in urine specimens (13 studies, 1,199 specimens). On the contrary, PTec-ET yielded a lower sensitivity of $50 \%$ for CSF and $77.8 \%$ for urine specimens with $100 \%$ specificity in our study. A previous study also showed a sensitivity of only $40 \%$ for the detection of M. tuberculosis by PTec-ET in CSF specimens using TB diagnosis as reference [21]. Other studies using PTec-ET test have either used very few CSF specimens $(n=2)[18]$ or the data were not tabulated for individual specimen types [27]. Taken together, our data showed higher sensitivity of Xpert than PTec-ET for the rapid diagnosis of EPTB, particularly in clinical specimens with histopathological evidence of TB and for the diagnosis of TB meningitis.

The Xpert also detected resistance to rifampicin in 1 specimen which is lower than the reported prevalence $(<1 \%)$ of MDR-TB in extrapulmonary specimens in $\mathrm{Ku}-$ wait [9]. However, it may be more useful for settings where MDR-TB is more prevalent, particularly in countries with a high burden of MDR-TB [1]. Both Xpert and PTec-ET tests have recently been updated as Xpert MTB/ RIF Ultra and BD Max ${ }^{\mathrm{TM}}$ MDR-TB, respectively [28, 29]. A preliminary evaluation of these 2 tests, mostly in respiratory specimens, found Xpert MTB/RIF Ultra to be more useful than $\mathrm{BD} \mathrm{Max}^{\mathrm{TM}} \mathrm{MDR}-\mathrm{TB}$ for the diagnosis of pulmonary TB in patients with paucibacillary load [30], it will be interesting to perform large scale evaluation of these 2 rapid tests for the diagnosis of EPTB and its resistance to rifampicin (and MDR-TB) in different settings.

\section{Conclusions}

The data reported in this study have shown that Xpert detected EPTB in significantly more specimens compared to PTec-ET in both MGIT 960 system culture-positive samples as well as in specimens that yielded culture and/or presented with histopathological evidence of TB (CRS). These differences were particularly pronounced in AFB-negative/culture-positive and AFB-negative/culture-negative/histopathology-positive samples. The performance of Xpert was also uniformly superior compared to PTec-ET in nearly all categories of extrapulmonary specimens. More importantly, Xpert detected TB in all cases of culture-positive CSF specimens for rapid diagnosis of TB meningitis, while the performance of PTec-ET was markedly lower. Thus, Xpert is superior to PTec-ET for the rapid diagnosis of EPTB.

\section{Statement of Ethics}

This study was approved by the Health Sciences Center Ethics Committee, Faculty of Medicine, Kuwait University (Approval no. VDR/EC/2947 dated December 12, 2016).

\section{Conflict of Interest Statement}

The authors have no conflicts of interest to declare.

\section{Funding Sources}

This study was supported in part by Kuwait University Research Sector grant MI 03/16.

\section{Author Contributions}

E.M., S.A., and H.S.E. designed the study. H.S.E. performed the experiments and collected the data. E.M. and S.A. analyzed the data and wrote the first draft of the manuscript. All the authors edited and approved the final version of the manuscript.

\section{References}

1 World Health Organization. Global tuberculosis report 2020. Geneva, Switzerland: WHO; 2020. Available from: https://www.who.int/ tb/publications/global_report/en/.

2 Ahmad S, Mokaddas E. Current status and future trends in the diagnosis and treatment of drug-susceptible and multidrug-resistant tuberculosis. J Infect Public Health. 2014;7(2): 75-91.
3 Dheda K, Gumbo T, Maartens G, Dooley KE, McNerney R, Murray M, et al. The epidemiology, pathogenesis, transmission, diagnosis, and management of multidrug-resistant, extensively drug-resistant, and incurable tuberculosis. Lancet Respir Med. 2017;5:291-360.

4 Walzl G, McNerney R, du Plessis N, Bates M, McHugh TD, Chegou NN, et al. Tuberculosis: advances and challenges in development of new diagnostics and biomarkers. Lancet Infect Dis. 2018;18(7):e199-210.
5 Ahmad S. New approaches in the diagnosis and treatment of latent tuberculosis infection. Respir Res. 2010;11:169.

6 Lee JY. Diagnosis and treatment of extrapulmonary tuberculosis. Tuberc Respir Dis. 2015;78(2):47-55.

7 Ahmad S, Mokaddas E, Al-Mutairi NM. Prevalence of tuberculosis and multidrug resistant tuberculosis in the Middle East Region. Expert Rev Anti Infect Ther. 2018;16(9):709-21. 
8 Ahmad S, Mokaddas E. Diversity of nontuberculous mycobacteria in Kuwait: rapid identification and differentiation of Mycobacterium species by multiplex PCR, INNOLiPA Mycobacteria v2 assay and PCR-sequencing of rDNA. Med Princ Pract. 2019;28: 208-15.

9 Mokaddas E, Ahmad S, Samir I. Secular trends in susceptibility patterns of Mycobacterium tuberculosis isolates in Kuwait, 19962005. Int J Tuberc Lung Dis. 2008;12:319-25.

10 Al-Ghafli H, Varghese B, Enani M, Alrajhi A, Al Johani S, Albarrak A, et al. Demographic risk factors for extra-pulmonary tuberculosis among adolescents and adults in Saudi Arabia. PLoS One. 2019;14(3):e0213846.

11 Ates Guler S, Bozkus F, Inci MF, Kokoglu OF, Ucmak H, Ozden S, et al. Evaluation of pulmonary and extrapulmonary tuberculosis in immunocompetent adults: a retrospective case series analysis. Med Princ Pract. 2015;24: 75-9.

12 Tyagi S, Sharma N, Tyagi JS, Haldar S. Challenges in pleural tuberculosis diagnosis: existing reference standards and nucleic acid tests. Future Microbiol. 2017;12:1201-18.

13 Bates M, Zumla A. The development, evaluation and performance of molecular diagnostics for detection of Mycobacterium tuberculosis. Expert Rev Mol Diagn. 2016;16(3):30722.

14 Denkinger CM, Schumacher SG, Boehme CC, Dendukuri N, Pai M, Steingart KR. Xpert MTB/RIF assay for the diagnosis of extrapulmonary tuberculosis: a systematic review and meta-analysis. Eur Respir J. 2014;44(2):43546.

15 Maynard-Smith L, Larke N, Peters JA, Lawn SD. Diagnostic accuracy of the Xpert MTB/ RIF assay for extrapulmonary and pulmonary tuberculosis when testing non-respiratory samples: a systematic review. BMC Infect Dis. 2014;14:709.
16 Kohli M, Schiller I, Dendukuri N, Dheda K, Denkinger CM, Schumacher SG, et al. Xpert ${ }^{\circ}$ MTB/RIF assay for extrapulmonary tuberculosis and rifampicin resistance. Cochrane Database Syst Rev. 2018;8:CD012768.

17 Boehme CC, Nabeta P, Hillemann D, Nicol MP, Shenai S, Krapp F, et al. Rapid molecular detection of tuberculosis and rifampin resistance. N Engl J Med. 2010;363(11):1005-15.

18 Alnimr AM, Hassan MI. Potential of two nucleic acid amplification assays for quantifying mycobacterial load in respiratory and non-respiratory specimens: a prospective study. Diagn Microbiol Infect Dis. 2014;78(3):237-41.

19 Ahmad S, Mokaddas E, Al-Mutairi N, Eldeen HS, Mohammadi S. Discordance across phenotypic and molecular methods for drug susceptibility testing of drug-resistant Mycobacterium tuberculosis isolates in a low TB incidence country. PLoS One. 2016;11(4): e0153563.

20 Mokaddas E, Ahmad S, Eldeen HS, Al-Mutairi N. Discordance between Xpert MTB/RIF assay and Bactec MGIT 960 culture system for detection of rifampin-resistant Mycobacterium tuberculosis isolates in a country with a low tuberculosis (TB) incidence. J Clin Microbiol. 2015;53(4):1351-4.

21 McHugh TD, Pope CF, Ling CL, Patel S, Billington $\mathrm{OJ}$, Gosling RD, et al. Prospective evaluation of BDProbeTec strand displacement amplification (SDA) system for diagnosis of tuberculosis in non-respiratory and respiratory samples. J Med Microbiol. 2004; 53(Pt 12):1215-9.

22 Ahmad S, Fares E, Araj GF, Chugh TD, Mustafa AS. Prevalence of S315T mutation within the katG gene in isoniazid-resistant clinical Mycobacterium tuberculosis isolates from Dubai and Beirut. Int J Tuberc Lung Dis. 2002;6:920-6.

23 Al-Mutairi N, Ahmad S, Mokaddas E. Performance comparison of four methods for rapid detection of multidrug-resistant Mycobacterium tuberculosis strains. Int J Tuberc Lung Dis. 2011;15:110-5.

24 Landis JR, Koch GG. The measurement of observer agreement for categorical data. Biometrics. 1977;33(1):159-74.

25 Tortoli E, Russo C, Piersimoni C, Mazzola E, Dal Monte P, Pascarella M, et al. Clinical validation of Xpert MTB/RIF for the diagnosis of extrapulmonary tuberculosis. Eur Respir J. 2012;40(2):442-7.

26 Karadağ A, Usta E, Bilgin K, Güney AK, Eroğlu C, Günaydın M. Comparison of culture, real-time DNA amplification assay and Ehrlich-Ziehl-Neelsen for detection of Mycobacterium tuberculosis. Balkan Med J. 2013; 30(1):13-5.

27 Bicmen C, Karaman O, Gunduz AT, Erer OF, Coskun M, Kaftan O, et al. Five years' evaluation of the BD ProbeTec system for the direct molecular detection of Mycobacterium tuberculosis complex in respiratory and nonrespiratory clinical samples. Pol J Microbiol. 2015; 64(4):391-4.

28 Osei Sekyere J, Maphalala N, Malinga LA, Mbelle NM, Maningi NE. A comparative evaluation of the new Genexpert MTB/RIF Ultra and other rapid diagnostic assays for detecting tuberculosis in pulmonary and extra pulmonary specimens. Sci Rep. 2019;9(1): 16587.

29 Ciesielczuk H, Kouvas N, North N, Buchanan R, Tiberi S. Evaluation of the BD MAX MDR-TB assay in a real-world setting for the diagnosis of pulmonary and extra-pulmonary TB. Eur J Clin Microbiol Infect Dis. 2020 39(7):1321-7.

30 Mokaddas EM, Ahmad S, Eldeen HS. GeneXpert MTB/RIF is superior to BBD Max MDRTB for diagnosis of tuberculosis (TB) in a country with low incidence of multidrug-resistant TB (MDR-TB). J Clin Microbiol. 2019; 57(6):e00537-19. 